\title{
Ultrastruktur der Haftapparate von Nematoplana coelogynoporoides (Turbellaria, Proseriata)
}

\author{
B. SOPOTT-EHLERS \\ II. Zoologisches Institut und Museum der Universität Göttingen; \\ D-3400 Göttingen, Bundesrepublik Deutschland
}

\begin{abstract}
Ultrastructure of the adhesive systems in Nematoplana coelogynoporoides (Turbellaria, Proseriata). The adhesive systems of Nematoplana coelogynoporoides Meixner, which are almost indiscernible in live animals, were examined with scanning and transmission electron microscopes. There are conformities with the fine structure of the adhesive systems in other Proseriata. However, there are also some special characteristics: the position of the adhesive systems in the epidermis; the number of anchor cells pro adhesive system; and the coexistence of intra-epithelial and insunk anchor cells in each adhesive system.
\end{abstract}

\section{EINLEITUNG}

Die Ausbildung von epidermalen Haftapparaten als Adaptation an den Lebensraum des Sandlückensystems ist von zahlreichen interstitiellen Organismen bekannt (vgl. Ax, 1963).

Erste Mitteilungen über die Ultrastruktur von Haftorganen, bei denen ein Zweidrüsensystem existiert, gibt Boaden (1968). Außer bei Gastrotrichen (vgl. auch Rieger et al., 1974; Teuchert, 1977) tritt ein Zweidrüsensystem auch bei Archianneliden (Martin, 1978) und in mehreren Turbellarien-Ordnungen (Tyler, 1976, 1977; Martin, 1978; Reuter, 1978) auf, u. a. auch bei den Proseriata. Die Vertreter dieser Ordnung haben gewöhnlich auf Grund zahlreicher über die Epidermisoberfläche hinausragender Papillen ein sehr gutes Haftvermögen. Obwohl dieses auch Nematoplana coelogynoporoides Meixner, 1938 in hohem Maße zu eigen ist, sind an lebenden Tieren keine prominenten Haftorgane zu erkennen. Auch lichtmikroskopische Schnittserien liefern nur unzureichende Informationen über die Natur des Anheftungsmechanismus dieser Art. Daher wurden raster- und transmissions-elektronenmikroskopische Untersuchungen durchgeführt, um den Feinbau der Haftapparate dieser Species aufzuklären.

\section{MATERIAL UND METHODE}

Aus marinen Sedimenten der Nordseeinsel Sylt wurden die Individuen mit der Seewassereis-Technik nach Uhlig extrahiert. 
Für die Raster-Elektronenmikroskopie wurden die Tiere entweder in 2,5\%igem Glutaraldehyd in $0,1 \mathrm{~m}$ Natriumcacodylat $\left(\mathrm{pH} \mathrm{7,2)}\right.$ bei $4{ }^{\circ} \mathrm{C} 2 \mathrm{~h}$ fixiert, mehrfach im Puffer gespuilt und mit $1 \%$ igem $\mathrm{OsO}_{4} 1 \frac{1 / 2}{\mathrm{~h}}$ nachfixiert oder die Fixierung erfolgte direkt in $1 \%$ igem Osmiumtetroxid in $0,1 \mathrm{~m}$ Natriumcacodylat ebenfalls bei $4{ }^{\circ} \mathrm{C}$. Entwässert wurde über eine aufsteigende Alkoholreihe, getrocknet mit $\mathrm{CO}_{2}$ in einem Critical-Point-Gerät Bomar SPC-900 EX. Das getrocknete Material wurde mit Ubertragungsfolie auf Aluminiumträgern befestigt und in einem Edwards Sputter Coater S 150 mit einer Goldschicht versehen. Sechzehn Träger mit jeweils 10-15 Tieren wurden an einem Zeiss Novascan 30 untersucht.

Da für die Transmissions-Elektronenmikroskopie weder eine einfache GlutaraldehydOsmiumfixierung noch eine Fixierung, bei der zur Erhöhung der Osmolarität Saccharose zugesetzt wurde, brauchbare Ergebnisse lieferte, wurden die Tiere in mit destilliertem Wasser im Verhältnis $3: 1$ verdünntes Seewasser (330 mOsm) gebracht und in diesem $48 \mathrm{~h}$ gehältert. Sodann erfolgte eine Fixierung in 2,5\%igem Glutaraldehyd in 0,1 m Natriumcacodylat wie oben beschrieben. Die Entwässerung wurde mit Aceton durchgeführt. Als Einbettungsmittel diente Araldit. Von acht Haftorganen wurden Ultradünnschnitte an einem Reichert OmU3 angefertigt, mit Uranylacetat und Bleicitrat kontrastiert und an einem Zeiss EM 9 S-2 untersucht.

\section{ERGEBNISSE}

Bei der lichtmikroskopischen Betrachtung von lebenden Nematoplana coelogynoporoides lassen sich zeitweilig postpharyngeal an den Körperseiten der Tiere kleine, grubenförmige Vertiefungen beobachten. Diese sind auch an lichtmikroskopischen Querschnittserien zu finden und zeigen nach Anwendung von Eisenhämatoxylin-Eosin-Färbung im Gegensatz zur übrigen, rot tingierten Epidermis einen graubraunen Farbton.

Am raster-elektronenmikroskopischen Abbild fallen bereits bei schwacher Vergrößerung lateral am Schwanzende kreisförmige Aussparungen von 25-30 $\mu \mathrm{m}$ Durchmesser (Abb. 1) in der einheitlichen Körperbewimperung auf. Diese Differenzierungen sind in gleichmäßigen Abständen angeordnet und ziehen um die Schwanzspitze herum (Abb. $1 \mathrm{~A}$ ), so daß sie einen U-förmigen Bogen bilden. Die Anzahl ist von der Körperlänge der Tiere abhängig. Maximal wurden 32 dieser Felder gezählt.

Bei stärkerer Vergrößerung erweisen sich diese kreisförmigen cilienfreien Areale als Bereiche, in denen in dichter Anordnung Mikrovilli stehen; außerdem können einzelne Sekretgranula unterschiedlicher Größe auftreten (Abb. 2 A, 3 B). In einigen Arealen sind Cilien innerhalb des Mikrovillifeldes zu erkennen (Abb. 3 B, 4 D).

Tyler (1976, p. 6) hebt als wesentliches Charakteristikum der Haftorgane die Zusammensetzung aus 3 Zelltypen hervor. Eine modifizierte Epidermiszelle, die Ankerzelle, wird von den Ausführgängen zweier Drüsenzellen durchzogen. Eine dieser Drüsenzellen produziert dunkle Sekretgranula und wird als Haftdrüse angesprochen, die andere sezerniert kleinere helle Sekrete und wird als Ablösedrüse bezeichnet. Im folgenden wird die Terminologie Tylers übernommen.

Betrachtet man Ultradünnschnitte, so erscheinen die grubenförmigen Vertiefungen bereits in der Ubersichtsvergrößerung als besonders strukturierte Epidermisbezirke (Abb. 

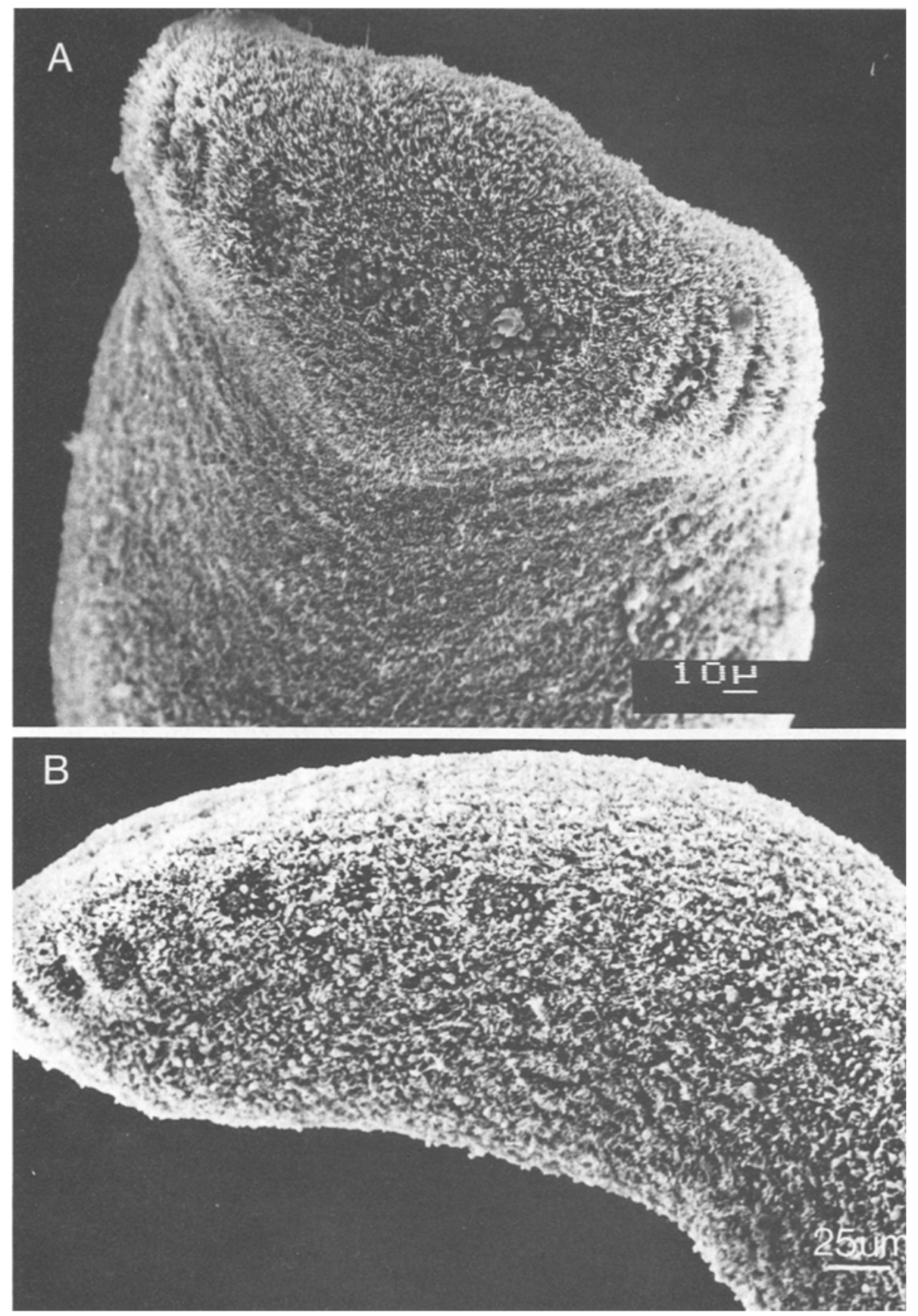

Abb. 1: Nematoplana coelogynoporoides. (A) Aufsicht auf das Schwanzende mit Haftapparaten. (B) Seitenansicht des Schwanzendes mit Haftapparaten 

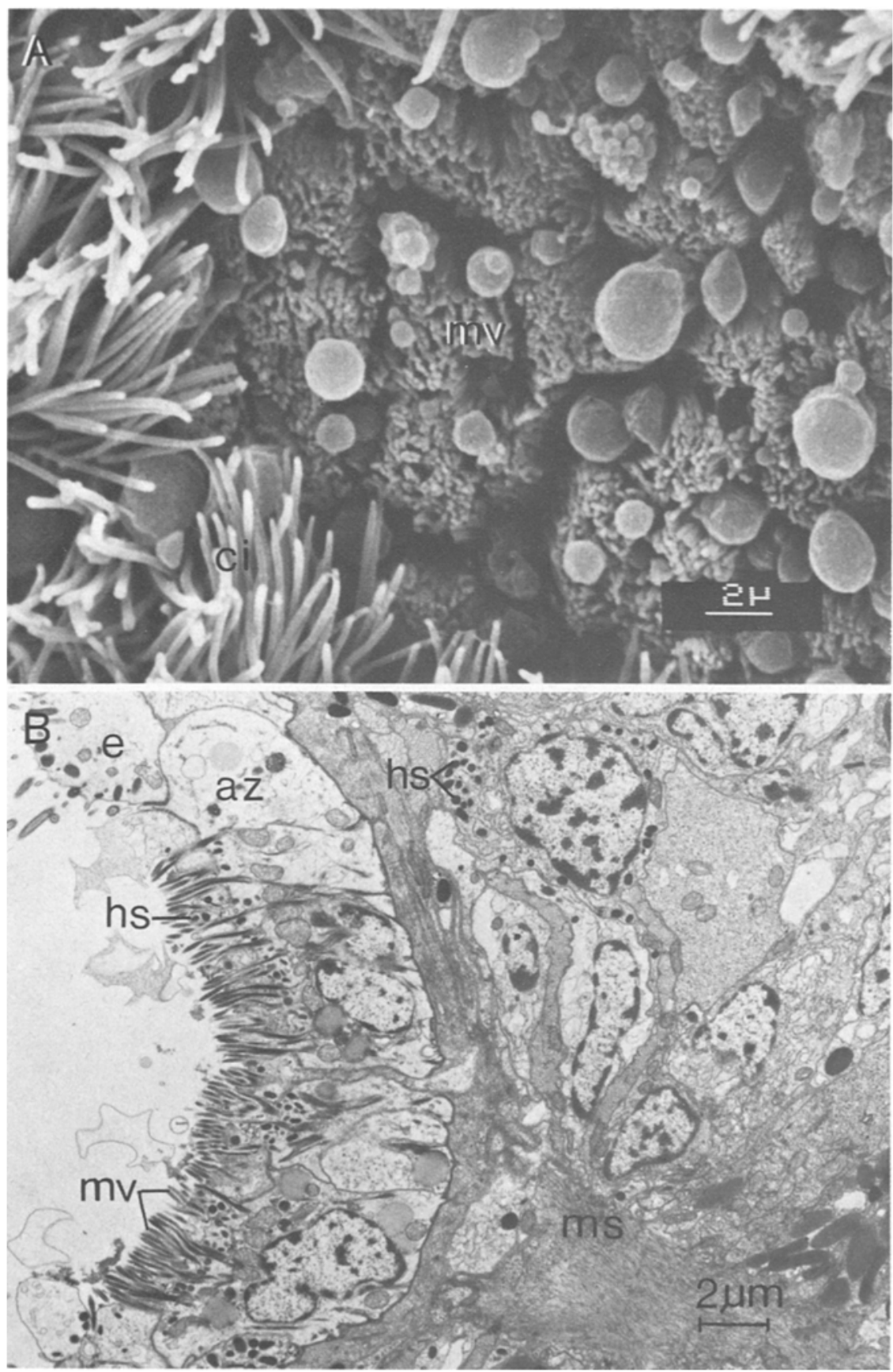

Abb. 2: Nematoplana coelogynoporoides. (A) REM-Aufnahme eines Haftorganes. (B) Peripherer Schnitt durch ein grubenförmig eingesenktes Haftorgan mit Muskelstrang 

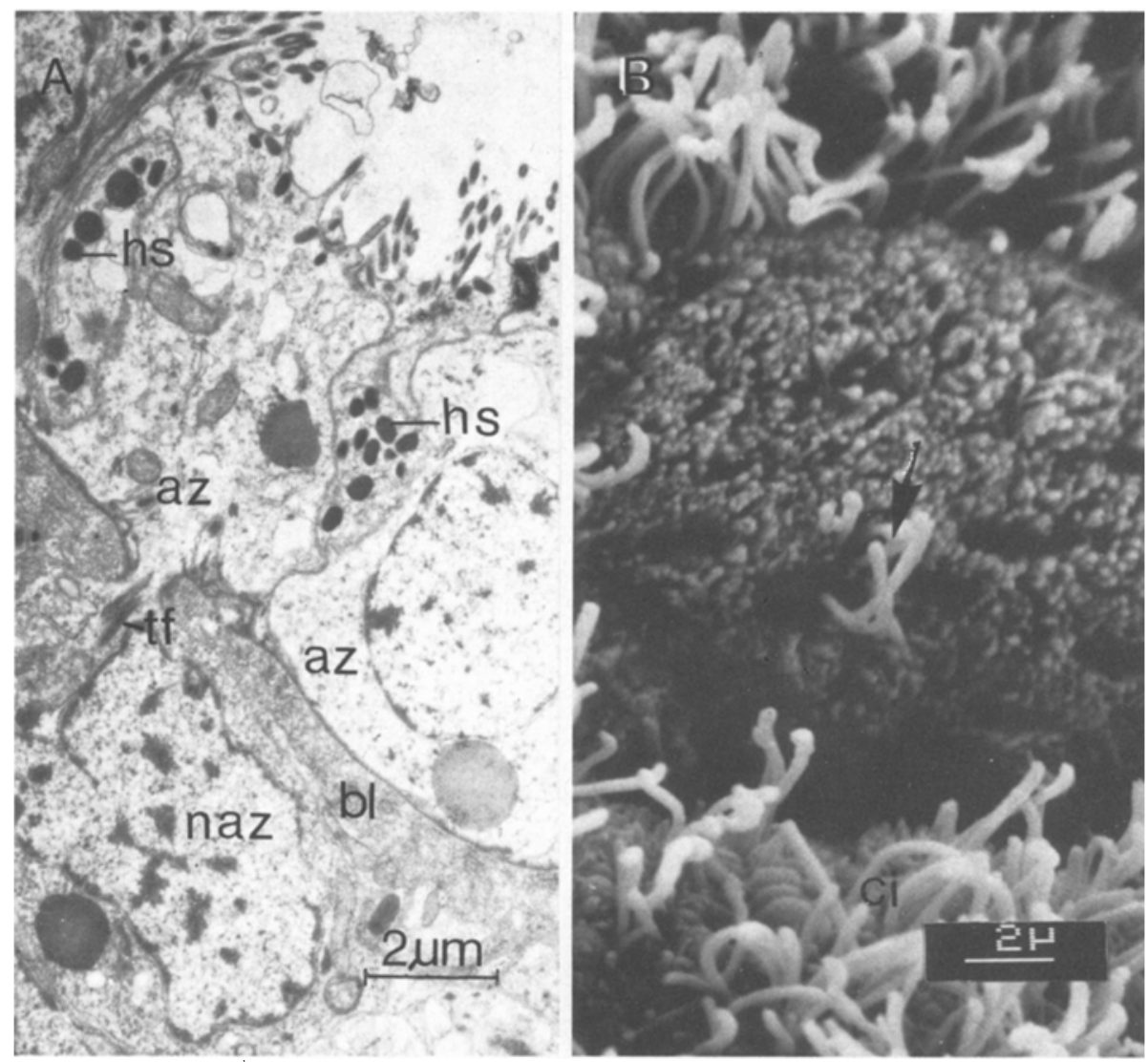

Abb. 3: Nematoplana coelogynoporoides. (A) Ankerzelle mit versenktem Kern. (B) Sensorische Cilien (Pfeil) zwischen den Mikrovilli eines Haftorgans

2 B, 4 A). Diese bestehen aus etwa sechs Zellen, den Ankerzellen. Die Kerne dieser Zellen liegen wie die der angrenzenden Epidermiszellen vorwiegend intraepithelial. Die freie Oberfläche weist sehr lange, dunkle Mikrovilli auf. Die dunkle Tönung der Mikrovilli wird durch eine starke Einlagerung von elektronendichtem, fibrösem Material, den Tonofilamenten, bewirkt. Diese durchlaufen basalwärts den gesamten Zellkörper und haben Kontakte zur Basallamina. Vereinzelt zieht das fibröse Material jedoch durch eine Unterbrechung in der Basallamina bis in tiefere Regionen. Stets wird dann auch ein Zellkern subepidermal beobachtet (Abb. 3 A). Es treten also neben Ankerzellen mit intraepithelial gelegenen Zellkernen auch solche mit versenkten Kernen auf.

Die unbewimperten Ankerzellen werden von Drüsenausführgängen durchdrungen, die zwei unterschiedliche membrangebundene Sekretgranula enthalten. Die dunklen ca. 0,25 $\mu$ großen Sekrettropfen werden als Produkte der Anheftungsdrüse (Abb. 2 B, 4 A, $4 \mathrm{~B}$ ), die helleren Sekrete mit einem Durchmesser von ca. 0,1 $\mu$ als Produkte der Ablösedrüse angesehen. Die Ausführgänge der Drüsen verzweigen sich. Pro Ankerzelle sind stets 

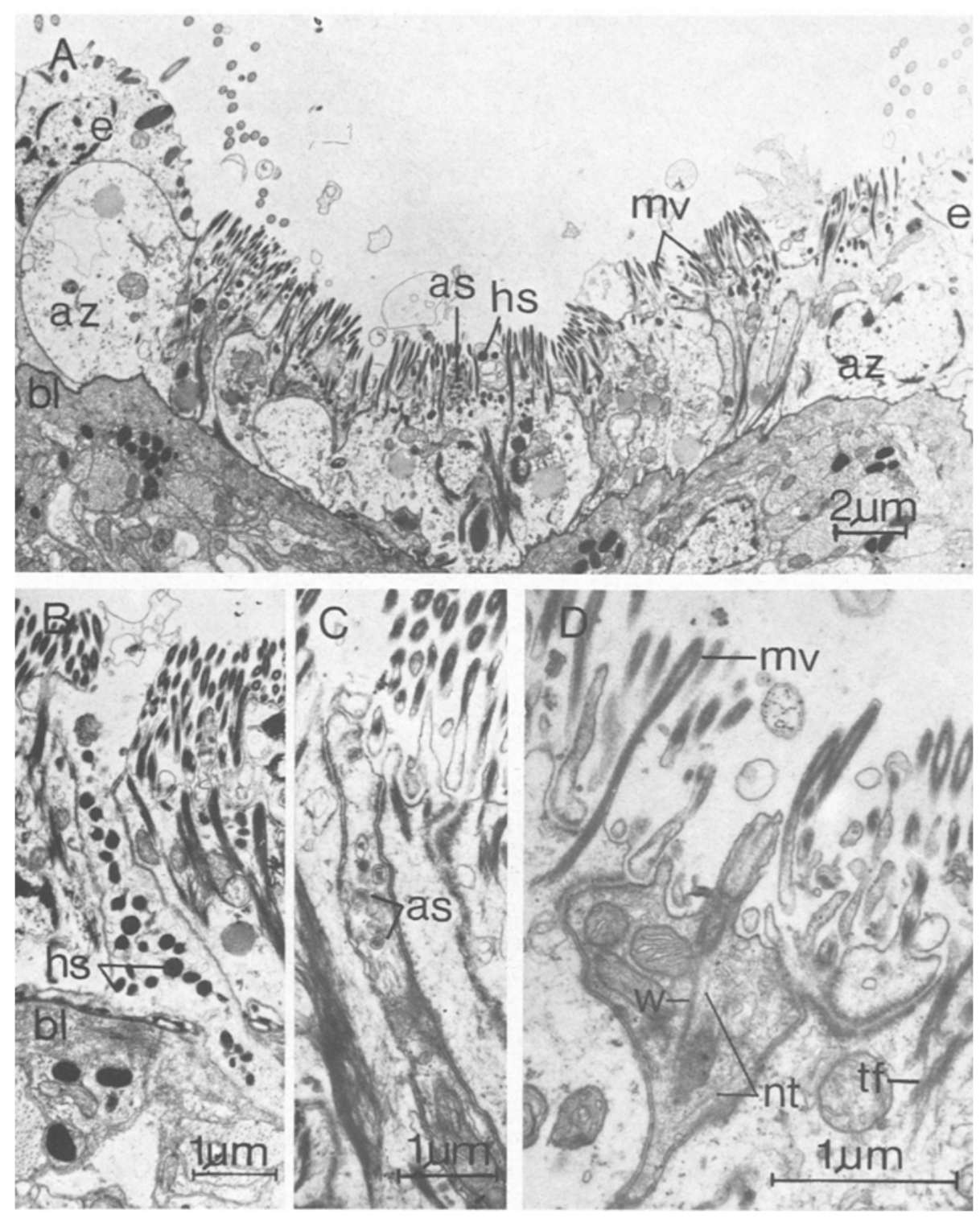

Abb. 4: Nematoplana coelogynoporoides. (A) Medianer Schnitt durch ein Haftorgan. (B) Ausführgang einer Haftdrüse. (C) Ausführgang einer Ablösedrüse. (D) Cilientragende Sinneszelle

mehr Ausführkanäle der Haftdrüse (Abb. 4 A, 4 C) als Kanäle der Ablösedrüse vorhanden. Die Mündungen der Ausführkanäle der Haftdrüse werden von einem Kranz der dunklen Mikrovilli umgeben. Zwar treten die Ausführgänge der Ablösedrüse auch zwischen diesen Mikrovilli an die Oberfläche, eine deutliche Zuordnung zu einem Mikrovillikranz ist jedoch nicht zu erkennen. Die Ausführgänge beider Drüsentypen enthalten zahlreiche 

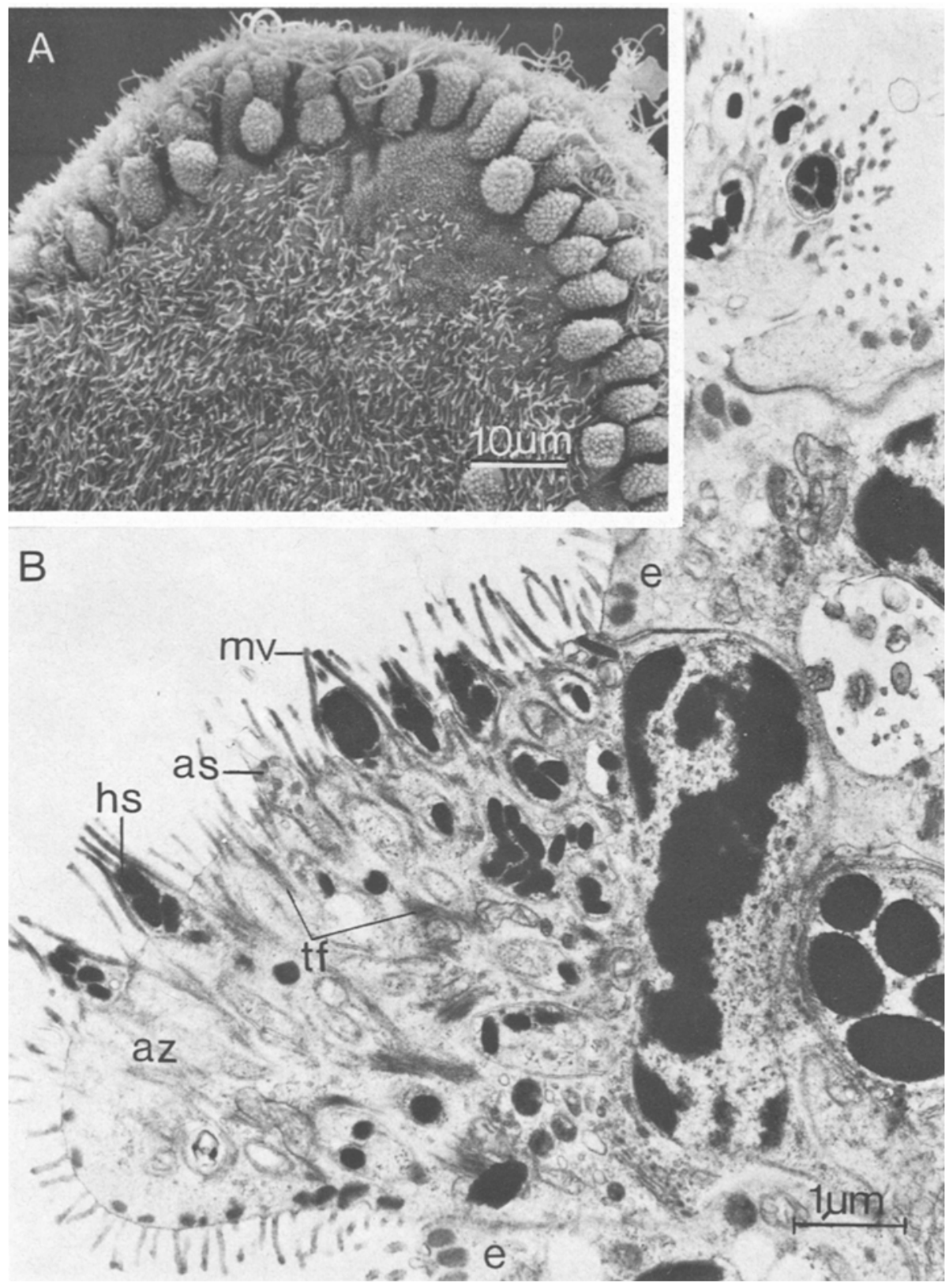

Abb. 5: Coelogynopora axi. Über die Epidermisoberfläche hinausragende Haftorgane. (A) Aufsicht auf die Haftorgane der Ventralseite. (B) Schnitt durch eine Ankerzelle 
Mikrotubuli, Ribosomen und Glykogen. Zuweilen werden auch Mitochondrien beobachtet (Abb. 4 A, 4 B). Die eigentlichen Drüsenkörper liegen unter dem Hautmuskelschlauch.

Die modifizierten Epidermiszellen werden neben den Ausführgängen der Drüsen von cilientragenden Sinneszellen durchdrungen (Abb. 3 B, 4 D). Diese unterscheiden sich von den Ankerzellen sowie von den unmodifizierten Epidermiszellen durch ein elektronendichteres, dunkles Plasma, das zahlreiche Neutrotubuli und lange, dunkle Mitochondrien enthält. Darüberhinaus tritt bei den Cilien der Sinneszellen nur eine einzige, senkrecht zur Körperoberfläche verlaufende Wurzel auf, während die Cilien des Deckepithels wie die anderer Proseriata eine parallel zur Oberfläche, mit der Spitze rostralwärts orientierte Hauptwurzel und eine senkrecht dazu stehende Nebenwurzel haben (vgl. Bedini \& Papi, 1974, p. 136, Fig. 22 C; Ehlers \& Ehlers, 1977, p. 198).

Die Verbindungen der Ankerzellen untereinander sowie die zwischen Ankerzellen und Drüsenausführgängen und Sinneszellen erfolgen durch Zonulae adhaerentes und septierte Desmosomen.

In der Nähe der Haftorgane verlaufen stets Längsnerven. Eine direkte Verbindung zwischen Haftorgan und Nerv konnte jedoch nicht nachgewiesen werden. Dorsal und ventral zweigen auf der Höhe der Haftorgane jeweils spezielle Muskelstränge (Abb. 2 B) von dem Hauptmuskelschlauch ab.

\section{DISKUSSION}

In wesentlichen Aspekten stimmt der Bau der Haftorgane von Nematoplana coelogynoporoides mit den von Tyler (1976) als typisch für die Proseriata hervorgehobenen Merkmalen überein. Unterschiede bestehen in folgenden Punkten:

(1) Lage der Haftorgane im Vergleich zur Epidermisoberfläche. Während die Haftorgane der übrigen Proseriata gewöhnlich deutlich über das Niveau des Deckepithels hinausragen, z. B. bei Coelogynopora axi Sopott (Abb. 5), sind die von N. coelogynoporoides grubenartig eingesenkt. Diese Erscheinung ist mit Sicherheit nicht artifiziell, da am lebenden Tier keine prominenten Hafteinrichtungen $\mathrm{zu}$ sehen sind und zudem bei der Präparation unterschiedliche Methoden angewandt wurden.

(2) Anzahl der Ankerzellen pro Haftorgan. Von allen bisher untersuchten Proseriata, d. h. einem Vertreter der Otoplaninae (Tyler, 1976), einer Vannuccia sp. (Tyler, unpubliziert), Monocelis cincta Karling (Martin, 1978), Botbriomolus balticus Meixner, Archilopsis unipunctata (Fabricius) (Reuter, 1978) sowie eigene Beobachtungen an 5 sicher determinierten Otoplaniden und den Coelogynoporiden Coelogynopora axi Sopott und Invenusta paracnida (Karling), weisen die Haftorgane von $N$. coelogynoporoides die größte Anzahl von Ankerzellen auf.

(3) Kernverhältnisse der Ankerzellen. Die Kerne des unmodifizierten Deckepithels von $N$. coelogynoporoides liegen stets intraepithelial. Im Bereich der Haftapparate nehmen die Kerne der Ankerzellen zumeist eine intraepitheliale Lage ein, doch treten regelmäßig auch einzelne Ankerzellen mit versenkten Kernen auf. Vergleichbare eigene Beobachtungen liegen bisher nur für Parotoplanina geminoducta Ax vor.

(4) Vorkommen sensorischer Cilien. Das Auftreten von cilientragenden Sinneszellen innerhalb eines Haftorganes war bei den bisher untersuchten Proseriaten-Arten unbekannt. 
(5) Muskelstränge. Tyler (1976, p. 45) gibt für Cicerina sp. an, daß die Haftgürtel des Vorderendes durch Kontraktion der Längsmuskulatur ausgestülpt werden. Die versenkte Lage der Haftorgane von $N$. coelogynoporoides macht eine entsprechende Funktion der beobachteten Muskelstränge sehr wahrscheinlich. Darüberhinaus können die Muskelstränge den bei einer Anheftung entstehenden Zugkräften entgegenwirken.

Die an Nematoplana coelogynoporoides beobachteten Besonderheiten der Haftorgane sprechen nicht gegen die von Tyler aus dem Aufbau der Haftvorrichtungen gezogenen Schlüsse über die Stellung der Proseriata im System der Turbellaria, zeigen aber, daß innerhalb der einzelnen Familien und Unterfamilien (vgl. auch Ax, 1956, p. 46) spezifische Variationen eines bestimmten Grundmusters auftreten können. Daher sind weitere eingehende vergleichende Untersuchungen an den Haftapparaten zur Aufklärung phylogenetischer Verwandtschaftsbeziehungen wünschenswert.

Danksagung. Der Akademie der Wissenschaften und der Literatur in Mainz danke ich für die großzügige Förderung der Untersuchungen durch Bereitstellung von Geräten für die Raster-Elektronenmikroskopie.

\begin{tabular}{llll} 
& \multicolumn{3}{c}{ Abkürzungen in den Abbildungen } \\
az & Ankerzelle & ms & Muskelstrang \\
as & Ablösesekret & mv & Mikrovilli \\
bl & Basallamina & naz & versenkter Kern einer Ankerzelle \\
ci & Cilien & nt & Neurotubuli \\
e & Epidermis & tf & Tonofilamente \\
hs & Haftsekret & w & Cilienwurzel
\end{tabular}

\section{ZITIERTE LITERATUR}

Ax, P., 1956. Monographie der Otoplanidae (Turbellaria). Morphologie und Systematik. Abh. Math.naturw. Kl. Akad. Wiss. Mainz. 13, 499-796.

- 1963. Die Ausbildung eines Schwanzfadens in der interstitiellen Sandfauna und die Verwertbarkeit von Lebensformcharakteren für die Verwandtschaftsforschung. Zool. Anz. 171, 51-76.

Bedini, C. \& Papi, F., 1974. Fine structure of the turbellarian epidermis. In: Biology of the Turbellaria. Ed. by N. W. Riser \& M. P. Morse. McGraw-Hill, New York, 108-147.

Boaden, P. J. S., 1968. Water movement - a dominant factor in interstitial ecology. Sarsia 34, 125-136.

Ehlers, U. \& Ehlers, B., 1977. Monociliary receptors in interstitial Proseriata and Neorhabdocoela (Turbellaria Neoophora). Zoomorphologie 86, 197-222.

Martin, G. G., 1978. The duo-gland adhesive system of the archiannelids Protodrilus and Saccocirrus and the turbellarian Monocelis. Zoomorphologie 91, 63-75.

Reuter, M., 1978. Scanning and transmission electron microscopic observations on surface structures of three turbellarian species. Zool. Scr. 7, 5-11.

Rieger, R., Ruppert, E., Rieger, G. E. \& Schoepfer-Sterrer, Ch., 1974. On the fine structure of gastrotrichs with the description of Chordodasys antennatus sp. n. Zool. Scr. 3, 219-237.

Teuchert, G., 1977. The ultrastructure of the marine gastrotrich Turbanella cornuta Remane (Macrodasyoida) and its functional and phylogenetical importance. Zoomorphologie 88, 189-246.

Tyler, S., 1976. Comparative ultrastructure of adhesive systems in the Turbellaria. Zoomorphologie 84, 1-76.

- 1977. Ultrastructure and systematics: an example for turbellarian adhesive organs. Mikrofauna Meeresboden 61, 271-286. 